\title{
Estimating Continuous 4D Wall Motion of Cerebral Aneurysms from 3D Rotational Angiography
}

\author{
Chong Zhang ${ }^{1,2}$, Mathieu De Craene ${ }^{2,1}$, Maria-Cruz Villa-Uriol ${ }^{1,2}$, \\ Jose M. Pozo ${ }^{1,2}$, Bart H. Bijnens ${ }^{3,1,2}$, and Alejandro F. Frangi ${ }^{1,2,3}$ \\ 1 Center for Computational Imaging \& Simulation Technologies in Biomedicine \\ (CISTIB), Universitat Pompeu Fabra, Barcelona, Spain \\ 2 Networking Research Center on Bioengineering, Biomaterials and Nanomedicine \\ (CIBER-BBN), Spain \\ 3 Institució Catalana de Recerca i Estudis Avançats (ICREA), Barcelona, Spain
}

\begin{abstract}
This paper presents a technique to recover dynamic 3D vascular morphology from a single 3D rotational X-ray angiography acquisition. The dynamic morphology corresponding to a canonical cardiac cycle is represented via a $4 \mathrm{D} B$-spline based spatiotemporal deformation. Such deformation is estimated by simultaneously matching the forward projections of a sequence of the temporally deformed 3D reference volume to the entire $2 \mathrm{D}$ measured projection sequence. A joint use of two acceleration strategies is also proposed: semi-precomputation of forward projections and registration metric computation based on a narrow-band region-of-interest. Digital and physical phantoms of pulsating cerebral aneurysms have been used for evaluation. Accurate estimation has been obtained in recovering sub-voxel pulsation, even from images with substantial intensity inhomogeneity. Results also demonstrate that the acceleration strategies can reduce memory consumption and computational time without degrading the performance.
\end{abstract}

\section{Introduction}

One 3D rotational X-ray angiography (3DRA) acquisition provides a sequence of $2 \mathrm{D}$ rotational X-ray angiographic images and an isotropic high-resolution 3D volumetric image reconstructed from them. As the acquisition lasts for a few seconds, the vascular motion occurring during the acquisition is captured in the 2D projections. However, since only a single volume is reconstructed, it does not provide the motion in $3 \mathrm{D}$. The objective of our work is to retrieve the dynamic 3D morphology of the imaged region from 3DRA by combining the volume, the projections, and an additionally synchronized physiological signal. The latter provides the temporal information of each projection in the cardiac cycles.

The application targeted in this paper is cerebral aneurysm wall motion estimation. Cerebral aneurysms are pathological dilations of brain arteries commonly located at the Circle of Willis. When they rupture, spontaneous subarachnoid hemorrhage usually follows, causing high morbidity and mortality rates [1]. 
It has been observed that aneurysms pulsate over the cardiac cycle 2 and that areas of higher pulsation coincide with the rupture sites [3]. However, the recovery of such pulsation is challenging because it is expected to be in the order of the voxel size. Also, existing aneurysm wall motion studies suffer from either strong artifacts [3] or partial motion estimation due to the use of a single projection view [45]. In our previous work [6], the aneurysm morphology at a given time instant is estimated from its temporal vicinity by matching projections of a deformed $3 \mathrm{D}$ reference volume to a sparse set of $2 \mathrm{D}$ projections in a weighted scheme. Similar to traditional gated reconstruction techniques, this approach approximates the spatiotemporal motion independently from one discrete time point to another, but fails to fully address the nature of motion: temporal consistency, that is, continuity over time.

In this paper, temporal consistency is achieved by formulating the inputs from $3 \mathrm{DRA}$ acquisitions into a $4 \mathrm{D}$ to multiple $2 \mathrm{D}$ image registration framework, which can be also found in [7. However, we propose to build a canonical cardiac cycle and then match the forward projections of the temporally deformed 3D reference volume to the $2 \mathrm{D}$ measured projection sequence. As a result, the dynamic morphology of cerebral aneurysms could be described continuous and smooth both spatially and temporally. However, the cost of memory storage and computational time for processing a high-resolution 4D image and two large 2D image sequences at the same time is very high. We therefore further introduce a joint use of two acceleration strategies: semi-precomputation of forward projections and registration metric computation based on a narrow-band region-of-interest (ROI).

\section{Algorithm Framework}

Building a Canonical Cardiac Cycle. During the rotation of the C-arm gantry, the total angular coverage of the measured projections during each cardiac cycle is $40-50^{\circ}$. This small range of the projection views may be less informative about the motion along certain directions. This could be compensated by introducing an additive constraint to the optimization process as in [7. We overcome this limitation by reordering all the projections into one canonical cardiac cycle. Thus the projection viewing angle range in any temporal vicinity is enriched, which subsequently ensures consistent 3D morphology estimation from all directions. An illustration of this process is shown in Fig. 1(a). We first normalize the period of each cardiac cycle to 1 , according to the synchronized physiological signal, e.g. electrocardiogram or pressure waveform. Hence any projection is acquired within the $[0,1)$ interval. All the $N$ projections are then sorted by this normalized time to build one canonical cardiac cycle as $\mathcal{I}=\left\{I_{t_{k}}(\mathbf{x}) \mid k=1 \ldots N, \mathbf{x} \in \mathcal{S}_{k} \subset \mathbb{R}^{3}\right\}$, where $I_{t_{k}}(\mathbf{x})$ represents the measured projection with the rotating field of view at projection plane $\mathcal{S}_{k}$ and at time $t_{k}$, $0 \leq t_{k} \leq t_{k+1}<1$.

4D to Multiple 2D Image Registration. As shown in Fig. 1(b), motion throughout the cardiac cycle is estimated by a $4 \mathrm{D}$ deformation $\mathcal{T}$ with parameters $\boldsymbol{\omega}$. And the 3D instantaneous morphology at time $t$ is given by: 


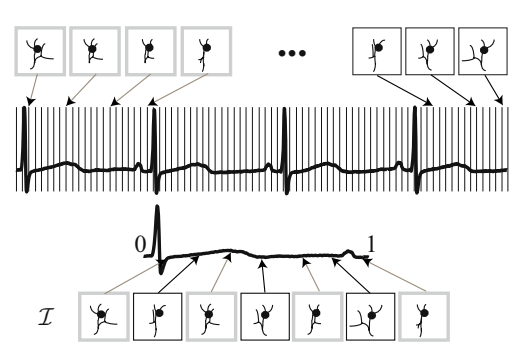

(a)

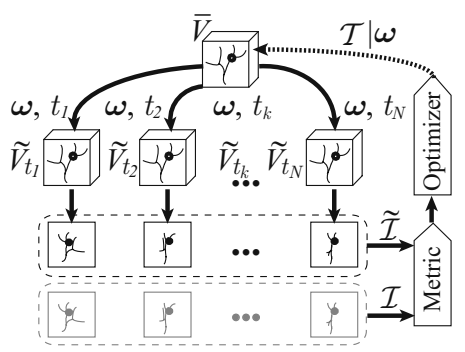

(b)

Fig. 1. (a) Building a canonical cardiac cycle. (b) 4D to multiple 2D image registration.

$$
\widetilde{V}_{t}(\mathbf{p})=\bar{V}(\mathcal{T}(\boldsymbol{\omega}, \mathbf{p}, t)),
$$

where $\mathbf{p}$ is a point on $\widetilde{V}_{t}$, and $\bar{V}$ stands for the reference volume. The deformation function $\mathcal{T}$ is chosen to be a $B$-spline based transformation [8]:

$$
\mathcal{T}(\boldsymbol{\omega}, \mathbf{p}, t)=\mathbf{p}+\sum_{\tau} \sum_{\mathbf{c}} \beta\left(\frac{t-t^{\tau}}{\Delta^{\tau}}\right) B\left(\frac{\mathbf{p}-\mathbf{p}^{\mathbf{c}}}{\Delta^{\mathbf{c}}}\right) \boldsymbol{\omega}^{\tau, \mathbf{c}},
$$

where $B(\cdot)$ is the $3 \mathrm{D}$ tensor product of $1 \mathrm{D}$ cubic $B$-spline functions $\beta(\cdot)$, defined on a sparse grid of $4 \mathrm{D}$ control points $\left(\mathbf{p}^{\mathbf{c}}, t^{\tau}\right)$, being $\mathbf{c}$ the spatial index and $\tau$ the temporal index, and $\left(\boldsymbol{\Delta}^{\mathbf{c}}, \Delta^{\tau}\right)$ the width of the functions in each dimension.

For each $I_{t_{k}}$ a corresponding forward projection $\tilde{I}_{t_{k}}$, also called digitally reconstructed radiograph (DRR), is generated to approximate the X-ray angiography. The pixel value $\tilde{I}_{t_{k}}(\mathbf{x})$ is calculated by integrating the voxel values of $\widetilde{V}_{t_{k}}$ along the ray from the $\mathrm{X}$-ray source $\mathbf{s}$ to $\mathbf{x}$ in the projection plane (see Fig. $2(\mathrm{a})$ ):

$$
\tilde{I}_{t_{k}}(\mathbf{x})=\int_{\mathcal{L}_{\mathbf{s}, \mathbf{x}}} \widetilde{V}_{t_{k}}(\boldsymbol{\gamma}) d \boldsymbol{\gamma} \approx \sum_{\mathbf{p}_{\ell} \in \mathcal{L}_{\mathbf{s}, \mathbf{x}}} \alpha_{\ell} \bar{V}\left(\mathcal{T}\left(\boldsymbol{\omega}, \mathbf{p}_{\ell}, t_{k}\right)\right)
$$

where $p_{\ell}$ denotes the $\ell$ th sampled point along $\mathcal{L}_{\mathbf{s}, \mathbf{x}}$, and $\alpha_{\ell}$ the sampling weight, which is the distance between two consecutive sampled points $\mathbf{p}_{\ell}$ and $\mathbf{p}_{\ell+1}$. Similarly, we denote $\tilde{\mathcal{I}}$ as the DRRs sequence, which is iteratively modified to match the measured projection sequence $\mathcal{I}$ for an optimal estimation with $\hat{\boldsymbol{\omega}}$ :

$$
\hat{\boldsymbol{\omega}}=\underset{\omega}{\operatorname{argmin}}\{M(\boldsymbol{\omega}, \mathcal{I}, \tilde{\mathcal{I}})\}
$$

where $M$ is the similarity metric between two mapping regions. The L-BFGSB algorithm [9] was used as the optimizer, and mutual information [10] as the metric function. Instead of computing the histograms individually for each projection pair, all sampled points from the entire sequence are considered as one region, thus forming a single histogram. That is, $M$ describes the similarity between two sequences. After obtaining $\hat{\boldsymbol{\omega}}$, we could substitute it into Eq. 1 to extract instantaneous motion at any given time instant. 


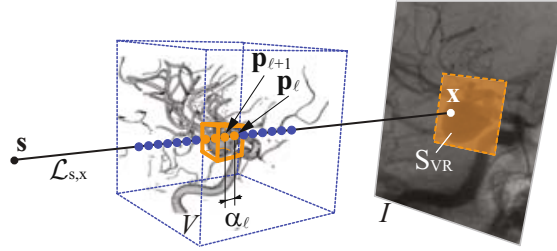

(a)

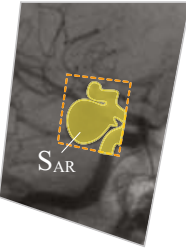

(b)

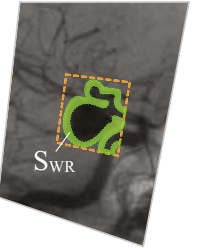

(c)

Fig. 2. (a) An illustration of DRRs computation. The sampling region $S_{V R}$ is the projected volume-of-interest. We introduce two object-adaptive sampling regions: (b) the aneurysm region $\mathrm{S}_{\mathrm{AR}}$ and (c) the aneurysm wall region $\mathrm{S}_{\mathrm{WR}}$.

\section{Acceleration Strategies}

Dealing simultaneously with a high-resolution 4D image and two large 2D image sequences, however, suffers from excessive memory requirements and computational load. For the method to be practically applicable, reducing both of them without degrading the performance is highly desirable. The following two joint strategies serve this purpose.

Semi-precomputed DRRs. A typical example of 3DRA patient data is shown in Fig. 2(a), where the structure of interest (i.e. aneurysm) is in the order of millimeters, occupying a very small part in the image. Thus, only this volume of interest (VOI) is deformed and the rest of the volume remains unmodified. On the other hand, in order to simulate X-ray projections, voxels of the entire volume must be integrated at each iteration to update forward projections. In order to avoid repeated computation, we precompute the voxel intensity integrals outside the VOI at each projection view. For instance in Fig. 2(a), the changing intensity sum of the sampled points on the ray $\mathcal{L}_{\mathbf{s}, \mathbf{x}}$ in the VOI is added at each iteration to the precomputed sum of the remaining ones for the pixel value at $\mathbf{x}$.

Narrow-Band Region-of-interest. Typically the projected VOI, denoted as $\mathrm{S}_{\mathrm{VR}}$ (Fig. 2(a)), is used as the sampling region to compute the metric. In this paper, we introduce two object-adaptive sampling regions: the projected aneurysm $\left(\mathrm{S}_{\mathrm{AR}}\right)$ and the projected aneurysm wall $\left(\mathrm{S}_{\mathrm{WR}}\right)$, shown in Fig. 2(b) and (c), respectively. Hence the image sequences in Eq. 4 are replaced by the sequences of these sampling regions. According to a typical histogram of a 3DRA cerebrovascular image, vessels belong to high intensity regions, among which the aneurysm region has even higher intensities [11. This property allows to roughly discriminate different regions based on intensity. $\mathrm{S}_{\mathrm{AR}}$ is obtained by first selecting a typical voxel value of the aneurysm dome, and then projecting only the points with voxel values larger than it. Similarly, $\mathrm{S}_{\mathrm{WR}}$ is obtained by taking the non-shared region of the two projected regions from typical voxel values of the background and the dome. Currently, we assume for each individual image the typical intensity values of the background and the aneurysm dome by sampling two small patches from these regions, respectively. Additionally, using $\mathrm{S}_{\mathrm{AR}}$ instead of $\mathrm{S}_{\mathrm{VR}}$ is to avoid possible influences of background structures on the estimation, and 
using $\mathrm{S}_{\mathrm{WR}}$ is to further avoid potential registration failures caused by intensity inhomogeneity at the projected aneurysm dome.

\section{Experiments and Results}

Phantom and Image Acquisition. A typical 3DRA flat panel system like Allura Xper FD20 (Philips Healthcare, Best, The Netherlands) acquires 120 Xray angiography images (resolution $1024^{2}$ pixels with $0.15^{2} \mathrm{~mm}^{2}$ /pixel), during a four-second $\mathrm{C}$-arm rotation of over 200 degrees. The images are then used to reconstruct a $3 \mathrm{D}$ volume of $256^{3}$ voxels at resolutions of $0.3^{3} \mathrm{~mm}^{3}$. In our experiments, 12 cases of digital phantom data were simulated to be consistent with these imaging settings using computer synthesized irregular geometries of dome diameter $=8,10$, and $12 \mathrm{~mm}$ and artery diameter $=4 \mathrm{~mm}$. Maximum pulsation amplitudes were $1 \%-4 \%$ of the dome diameter $(0.08-0.48 \mathrm{~mm})$. In order to simulate realistic 3DRA data, we fused the phantom data into a clinical dataset (Fig. 3). Details of this simulation are described in 6]. Physical phantom data was obtained with a silicone side-wall aneurysm phantom (Elastrat, Geneva, Switzerland), representing realistic dimensions of vascular structures in human bodies: dome diameter $=10 \mathrm{~mm}$ and artery diameter $=4 \mathrm{~mm}$. It was filled with contrast agent (Iomeron 400, Bracco Imaging SpA, Milan, Italy) and connected to a customized pulsatile pump. Images were acquired from an aforementioned Allura Xper FD20 imaging suite.

Results. Experiments were executed on an Intel @ Core ${ }^{\mathrm{TM}} 2$ Quad CPU Q6600 $2.40 \mathrm{GHz}$ with $4 \mathrm{~GB}$ of memory and no parallelization was used. All the experiments employed the semi-precomputed DRRs strategy, which essentially produced equal results but with a $3-4 \mathrm{x}$ speedup. This ratio is the proportion between the ray path length of the full volume and that of the VOI. The B-spline control point grid spacing was about $1.5 \mathrm{~mm}$ for the spatial dimensions, and $12.5 \%$ of the canonical cardiac cycle for the temporal dimension.

We first carried out digital phantom experiments for quantitative validation. A set of deformed volume images at discrete time points were extracted according to the estimated transformation. Then, a relative estimation error was measured at each time point $t$ as a percentage of the pulsation range: $\varepsilon(t)=\left(m_{r}(t)-\right.$ $\left.m_{g}(t)\right) / \hat{m}_{g} \times 100 \%$, where $m_{g}(t)$ is the ground-truth pulsation measurement (e.g., volume) at $t, m_{r}(t)$ the corresponding estimated measurement, and $\hat{m}_{g}$ the variation range of $m_{g}(t)$ over the canonical cycle. For each of the 12 cases in Fig. 3, a boxplot is shown, indicating the relative error in volume changes, $\varepsilon_{V}$, at 16 equally distributed time points. Aneurysm volume was measured using a similar strategy as in [12. Except for two tests with maximum pulsation less than $0.1 \mathrm{~mm}$, estimation errors were below $10 \%$.

The following experiment aimed at analyzing the sensitivity to spurious projection intensity variations. Although it is mainly caused by blood turbulence during contrast agent filling, the instantaneous local inhomogeneity is caused by multiple factors. We have synthesized such pattern based on a clinical dataset that presented substantial nonuniform contrast filling. For each of the digital 

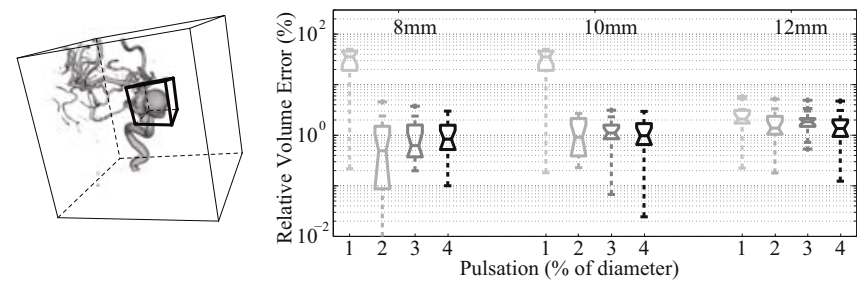

Fig. 3. Boxplots of $\varepsilon_{V}$ at 16 equally distributed time points for 12 digital phantom cases, in which a digital phantom is fused into a clinical dataset

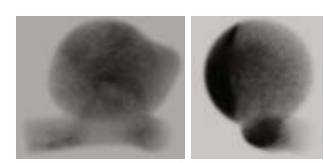

(a)

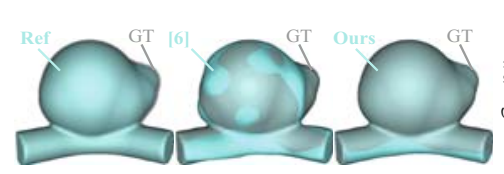

(b)

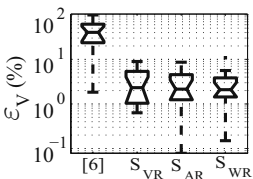

(c)

Fig. 4. (a) Projections with contrast inhomogeneity synthesized based on clinical data. (b) The ground-truth shape (GT) at one time instant overlaid with (from left to right): the reference shape (Ref), estimation using the method in [6], and our estimation. (c) Comparison of $\varepsilon_{V}$ between [6] and our technique with three sampling regions.

phantom projections, an image patch was taken from the aneurysm dome of one different clinical projection and texture-mapped to the projected phantom dome, see Fig. 4(a). Then, we performed motion estimation using our technique with regions $\mathrm{S}_{\mathrm{VR}}, \mathrm{S}_{\mathrm{AR}}$, and $\mathrm{S}_{\mathrm{WR}}$, and the method in our previous work [6]. As shown in Fig. 4(b) and (c), a relative volume error $\varepsilon_{V}$ below $10 \%$ was achieved using our technique, whereas the method in [6] failed to properly retrieve the motion. This suggests that our 4D smooth estimation is more robust to large image intensity inhomogeneity both temporally and spatially. Additionally, a slightly higher accuracy was obtained using the narrow-band ROI as most of the inhomogeneous regions did not contribute to the metric computation.

In terms of computational efficiency, we investigated the effects of using different sampling regions and angular resolutions along the C-arm gantry trajectory. An example for a phantom having 10-mm diameter and 3\% maximum pulsation is given in Fig. 5, where $\varepsilon_{V}$ is plotted together with the computational time for the same number of iterations. The angular resolution of the measured projection sequence was downsampled by a factor of 1-4. Results show similar accuracy $(<5 \%)$ achieved from the three sampling regions combined with the angular resolution downsampling factor up to 3 . However, the computational time can be proportionally reduced due to both of them. Compared to the computational time of 10-15 minutes for an estimation at a single time instant in our previous work [6, we could obtain the motion over the whole cycle in 15-20 minutes using $S_{W R}$ with the angular resolution downsampling factor of 3 . As a result, the use of the narrow-band ROI combined with a subsampled projection set could potentially save both memory and computational time without degrading the performance. 


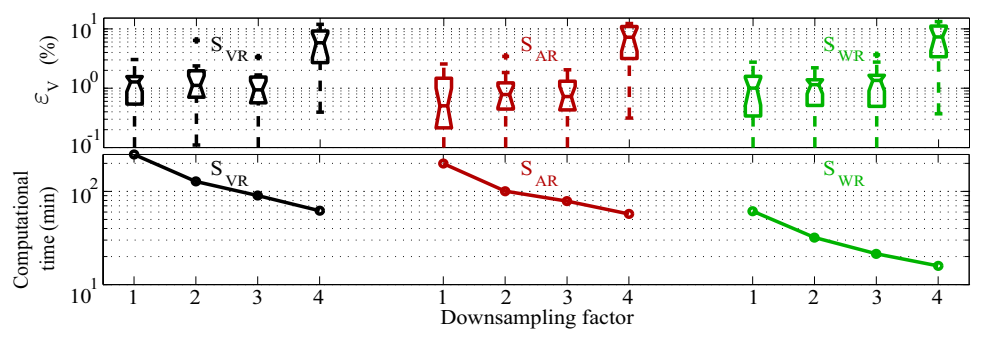

Fig. 5. Comparison in estimation error $\varepsilon_{V}$ and computational time using different sampling regions and angular resolution downsampling factors
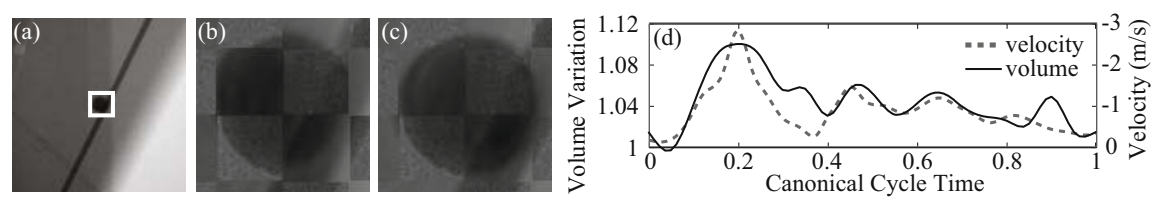

Fig. 6. (a) An X-ray projection image. (b,c) Checkerboard images of the measured and the forward projection from the reference volume and our estimation, respectively. (d) The volume variations along with the Doppler ultrasound velocity waveform.

We further tested the proposed technique on 3DRA data of the pulsating physical phantom acquired in a clinical environment. Due to the unknown groundtruth, results are qualitatively evaluated. As shown in Fig. 6, our estimation was able to correct the misalignment between the measured and the forward projections. The volume variation waveform was also compared to a Doppler ultrasound waveform measured at the parent vessel inlet. A similar pattern is observed between them, which is in agreement with the findings in [13.

\section{Discussion and Conclusions}

We have demonstrated on digital and physical phantoms the performance of the proposed technique in estimating continuous $4 \mathrm{D}$ cerebral aneurysm wall motion from 3DRA. Accurate estimation has been obtained in recovering subvoxel pulsation, even from images with substantial intensity inhomogeneity. The joint use of the acceleration strategies has shown a reduction of memory cost as well as computational time (3-4x speedup from semi-precomputation and $10 \mathrm{x}$ from narrow-band ROI), while preserving the accuracy of the motion estimation. These results suggest its feasibility towards estimating 4D aneurysmal wall motion from patient data. Finally, the fact that motion is estimated from a single 3DRA acquisition dataset implies no additional radiation risk for the patient, which facilitates its clinical take-up.

Acknowledgments. The authors thank Dr. D. Rüfenacht and L. Augsburger from Geneva University Hospitals, for providing the aneurysm silicone phantom 
and the pulsatile pump, and Dr. J. Blasco, Dr. J. Macho and Dr. E. Vivas for the 3DRA acquisitions of the physical phantom. This work was partially supported by Philips Healthcare, Best, The Netherlands, the CENIT-CDTEAM grant funded by the Spanish Ministry Of Science and Innovation (MICINNCDTI), and partially generated in the framework of the @neurIST Integrated Project, which is co-financed by the European Commission (IST-027703).

\section{References}

1. Brisman, J.L., Song, J.K., Newell, D.W.: Medical progress: cerebral aneurysms. N. Engl. J. Med. 355(9), 928-939 (2006)

2. Meyer, F.B., Huston III, J., Riederer, S.S.: Pulsatile increases in aneurysm size determined by cine phase-contrast MR angiography. J. Neurosurg. 78(6), 879-883 (1993)

3. Ishida, F., Ogawa, H., Simizu, T., Kojima, T., Taki, W.: Visualizing the dynamics of cerebral aneurysms with four-dimensional computed tomographic angiography. Neurosurgery 57(3), 460-471 (2005)

4. Dempere-Marco, L., Oubel, E., Castro, M., Putman, C., Frangi, A.F., Cebral, J.R.: CFD analysis incorporating the influence of wall motion: Application to intracranial aneurysms. In: Larsen, R., Nielsen, M., Sporring, J. (eds.) MICCAI 2006. LNCS, vol. 4191, pp. 438-445. Springer, Heidelberg (2006)

5. Oubel, E., De Craene, M., Putman, C., Cebral, J., Frangi, A.F.: Analysis of intracranial aneurysm wall motion and its effects on hemodynamic patterns. In: Proc. SPIE Med. Imag.: Physiology, Function, and Structure from Medical Images, p. 65112A (2007)

6. Zhang, C., Villa-Uriol, M.-C., De Craene, M., Pozo, J.M., Frangi, A.F.: Morphodynamic analysis of cerebral aneurysm pulsation from time-resolved rotational angiography. IEEE Trans. Med. Imaging 28(7), 1105-1116 (2009)

7. Zeng, R., Fessler, J.A., Balter, J.M.: Estimating 3-D respiratory motion from orbiting views by tomographic image registration. IEEE Trans. Med. Imaging. 26(2), 153-163 (2007)

8. Rueckert, D., Sonoda, L.I., Hayes, C., Hill, D.L.G., Leach, M.O., Hawkes, D.J.: Non-rigid registration using free-form deformations: Application to breast MR images. IEEE Trans. Med. Imaging 18(8), 712-721 (1999)

9. Zhu, C., Byrd, R.H., Nocedal, J.: L-BFGS-B: Algorithm 778: L-BFGS-B, FORTRAN routines for large scale bound constrained optimization. ACM Trans. Math. Software 23(4), 550-560 (1997)

10. Mattes, D., Haynor, D.R., Vesselle, H., Lewellen, T.K., Eubank, W.: PET-CT image registration in the chest using free-form deformation. IEEE Trans. Med. Imaging. 22(1), 120-128 (2003)

11. Bogunović, H., Radaelli, A.G., De Craene, M., Delgado, D., Frangi, A.F.: Image intensity standardization in 3D rotational angiography and its application to vascular segmentation. In: Proc. SPIE Med. Imag.: Image Processing, p. 691419 (2008)

12. Holden, M., Schnabel, J.A., Hill, D.L.G.: Quantification of small cerebral ventricular volume changes in treated growth hormone patients using nonrigid registration. IEEE Trans. Med. Imaging. 21(10), 1292-1301 (2002)

13. Löw, M., Perktold, K., Raunig, R.: Hemodynamics in rigid and distensible saccular aneurysms: a numerical study of pulsatile flow characteristics. Biorheology 30, 287298 (1993) 